\title{
Lower bone mineral density in Somali women living in Sweden compared with African-Americans
}

\author{
Taye Demeke • Gamal Abd El-Gawad • \\ Amra Osmancevic • Martin Gillstedt • \\ Kerstin Landin-Wilhelmsen
}

Received: 5 October 2014 / Accepted: 6 February 2015 /Published online: 19 February 2015

(C) The Author(s) 2015. This article is published with open access at Springerlink.com

\begin{abstract}
Summary Vitamin D deficiency can lead to osteomalacia. Bone mineral density was lower in Somali women, living in Sweden, in relation to both the American and the AfricanAmerican reference populations. The majority, $73 \%$, had vitamin D deficiency, and supplementation should be considered to prevent from osteomalacia, osteoporosis and future fractures.

Purpose Low vitamin D can lead to osteomalacia. The hypothesis was that bone mineral density (BMD) in Somali women living in Sweden was lower in comparison with different ethnic reference populations.

Methods Women from Somalia, $n=67$, median age 35.8 years (range 18 to 56 ), latitude $0-10^{\circ}$ North living in Gothenburg, Sweden, latitude $57^{\circ}$ North, $>2$ years were studied. All wore traditional Islamic clothing and had skin photo type V. BMD was recorded as the $Z$-score and compared with white American and African-American women, respectively, using
\end{abstract}

The abstract was presented at the Endocrine Society's Annual Meeting, Chicago, USA, June 2014

T. Demeke $(\bowtie)$

Hjällbo Primary Health care, S-424 32 Gothenburg, Sweden

e-mail: taye.demeke@vgregion.se

G. A. El-Gawad

Family Health Primary Health Care, Gothenburg, Sweden

A. Osmancevic $\cdot$ M. Gillstedt

Department of Dermatology, Sahlgrenska University Hospital,

Gothenburg, Sweden

K. Landin-Wilhelmsen

Section for Endocrinology, Institution of Medicine, Sahlgrenska University Hospital at Sahlgrenska Academy, University of

Gothenburg, Gothenburg, Sweden standard data from the dual energy X-ray absorptiometry (DXA) manufacturer (Lunar Prodigy enCORETM, GE Healthcare, LU44663). A fasting blood test was drawn for analysis of serum 25(OH)D.

Results The median Z-score compared with the American white population was $-0.9 \mathrm{SD}$ of the lumbar spine $(p<0.00001), 0.1 \mathrm{SD}$ of the left hip and $0.0 \mathrm{SD}$ of the right hip (ns).

The median $Z$-score compared with the African-American population was $-1.6 \mathrm{SD}$ of the lumbar spine $(p<0.00001)$, $-0.9 \mathrm{SD}$ of the left hip and $-0.9 \mathrm{SD}$ of the right hip $(p<0.001)$. The majority, $73 \%$, had vitamin D deficiency, serum $25(\mathrm{OH}) \mathrm{D}<25 \mathrm{nmol} / 1(<10 \mathrm{ng} / \mathrm{ml})$. BMD did not correlate to vitamin $\mathrm{D}$ levels or to the number of years in Sweden. One wrist fracture was reported.

Conclusions BMD was lower in these fairly young immigrant women from Somalia, living in Sweden, in relation to both the American and the African-American reference populations. Vitamin D supplementation should be considered to prevent from osteomalacia, osteoporosis and future fractures.

Keywords Bone mineral density · Vitamin D . African-American $\cdot$ Immigrants

\section{Introduction}

Vitamin D deficiency is considered to be an epidemic of worldwide proportion, involving all races and all age groups [1]. It is an important determinant of skeletal development and maintenance of bone mass throughout life [2]. Vitamin D promotes calcium absorption and bone mineralisation and is positively associated with bone mineral density (BMD) [3]. The level of vitamin $\mathrm{D}$ required to attain optimal bone health, 
however, is still unclear [4]. The existence of ethnic variations in BMD is recognized [5], but the underlying mechanism accounting for the differences is not yet fully understood. It is well established that African-Americans have lower fracture risk due to higher BMD compared with their white counterparts in the USA [6]. Somali women and Sudanese immigrants in the USA [7], despite sharing the same African heritage, exhibit low BMD which in part was ascribed to malnutrition in sub-Saharan Africa [8]. It appears that there exists a specific bone metabolic mechanism favouring blacks of the West African origin [9].

The negative health effects of vitamin D deficiency and low BMD in the immigrant minority population are not yet studied in Sweden. Thus, the purpose was to study BMD status of Somali women living in Sweden compared to African-American women and white American women in the USA using data supplied by the dual energy X-ray absorptiometry (DXA) manufacturer. Serum vitamin D levels were also measured. The aim was to test the hypothesis that BMD in Somali women living in Sweden was lower than that in the white and African-American reference population.

\section{Methods}

A total number of 67 Somali women from latitude $0-10^{\circ} \mathrm{N}$, age 18-56 years, living in Gothenburg, Sweden, latitude $57^{\circ}$ $\mathrm{N}$, since at least 2 years, were recruited on a voluntary basis via flyers to Somali community shops and placed advertisements at healthcare units, a nearby pharmacy and a super market. It is estimated to be around 4000 Somalis in Gothenburg with a total population of 500,000. Different Somali associations were contacted to inform about the objective of the study. All subjects willing to participate, $n=104$, were referred to our research assistant and were examined by an experienced physician (TD) before enrolment. DXA was performed in 67 women and 37 were lost to follow-up. All but 1 was premenopausal.

Weight $(\mathrm{kg})$ was measured wearing indoor clothing without shoes. Height $(\mathrm{cm})$ was measured using wall-mounted stadiometer without shoes. Body mass index (BMI) was calculated as a body weight divided by the height squared $\left(\mathrm{kg} / \mathrm{m}^{2}\right)$.

BMD measurements were made by DXA at the lumbar spine and the left and right hip. The coefficients of variation for GE/LUNAR device at the lumbar and femoral neck were 1.22 and 1.97. BMD was recorded as the $Z$-score (difference in SD from the mean of healthy, matched women). These results were adjusted for age, body weight, gender and skin type for both patients and referents. A $Z$-score of -2.0 or less signifies low BMD for age, www.ISCD.org. The BMD in Somali women was compared both with BMD in white American women and BMD in African-American women using standard data provided by the DXA manufacturer (LUNAR Prodigy enCORETM, GE Healthcare, LU44663). A fasting blood test was drawn for analysis of serum 25(OH)D (DiaSorin, Stillwater, MN, USA) and serum intact parathyroid hormone (S-PTH) (ABBOT, Architect, Wiesbaden, Germany). Photometry $600 \mathrm{~nm}$ was used to determine concentrations of S-calcium and bone-specific alkaline phosphatase (ALP). Ionized calcium in serum was measured using ion-selective electrodes.

Participants completed a questionnaire regarding recent medication both prescribed and none prescribed, parity, duration of residency in Sweden, smoking habits, previous diseases and fractures. Subjects also filled in a visual analogue scale (VAS), $0-10 \mathrm{~cm}$ (low to high), in order to evaluate pain. Quality of life, including physical and mental scores, was selfrecorded by Short Form (SF) -36, 0-100 (low to high), with questionnaires [10]. The survey was conducted in Gothenburg, during autumn and winter 2010, 2011 and 2012. Eligibility criteria for inclusion comprised no history of serious disease states, no medication that might interfere or affect bone metabolism, no pregnancies and no lactations.

A written consent was obtained, and the project was approved by the Swedish research ethical committee at the University of Gothenburg.

\section{Statistical analyses}

Mean, standard deviations and medians were calculated with conventional methods. Wilcoxon's signed rank test was used to test for differences between subject BMD values and the reference distribution. Correlations were performed with Spearman's test. $p<0.05$ was considered statistically significant.

\section{Results}

Anthropometric and background data, VAS, SF-36 scoring and BMD data are given in Table 1. The median value of $Z$ score compared with the reference interval for the American white population was $-0.9 \mathrm{SD}(\min -3.4, \max 1.6)$ of the lumbar spine $(p<0.00001), 0.1 \mathrm{SD}(\min -1.7, \max 2.1)$ of the left hip (ns) and $0.0 \mathrm{SD}(\min -2.0, \max 1.7)$ of the right hip (ns) (Fig. 1a, b).

The median value of $Z$-score compared with reference interval for the African-American population was -1.6 SD (min $-4.1, \max 0.9)$ of the lumbar spine $(p<0.00001),-0.9 \mathrm{SD}$ (min $-2.8, \max 1.0)$ of the left hip $(p<0.00001)$ and -0.9 SD (min $-3.0, \max 2.2)$ of the right hip $(p<0.00001)$ (Fig. 1c, d).

Eleven (16\%) and 26 (39\%) women had low BMD for age according to the American and African-American referents, 
Table 1 Characteristics of the 67 Somali women who performed bone mineral density measurement with dual energy Xray absorptiometry (DXA). Mean \pm SD and range are given for continuous variables

$S-A L P$ serum alkaline phosphatase, $S$ - $P T H$ serum intact parathyroid hormone, $V A S$ visual analogue scale

\begin{tabular}{llll}
\hline & Mean \pm SD & $\begin{array}{l}\text { Range } \\
\text { Min-max }\end{array}$ & $\begin{array}{l}\text { Number of patients } \\
\text { with data }\end{array}$ \\
\hline Age, years & $35.1 \pm 9.2$ & $18-55.9$ & 67 \\
Height, cm & $163.6 \pm 6.9$ & $151-182$ & 67 \\
Body weight, kg & $72.1 \pm 13.7$ & $39-99$ & 67 \\
BMI, kg/m ${ }^{2}$ & $27.0 \pm 5.1$ & $16.9-37.3$ & 67 \\
Years in Sweden & $14.5 \pm 6.2$ & $3-24$ & 65 \\
Parity, $n$ & $2.8 \pm 2.6$ & $0-11$ & 64 \\
Non-smokers, $n$ (\%) & $0(100 \%)$ & & 67 \\
Fractures, $n$ & 1 & $0-1$ & 67 \\
S-25(OH)D, nmol/1 & $22.4 \pm 13.0$ & $0-72$ & 67 \\
S-25(OH)D $<25$ nmol/l=deficiency, $n(\%)$ & $49(73.1 \%)$ & & 67 \\
S-calcium, mmol/1 & $2.32 \pm 0.09$ & $2.15-2.26$ & 67 \\
S-ionized calcium, mmol/1 & $1.23 \pm 0.04$ & $1.17-1.21$ & 67 \\
S-bone-specific ALP, $\mu$ kat/l & $14.7 \pm 5.6$ & $6.0-34.0$ & 67 \\
S-PTH, pmol/1 & $5.60 \pm 1.98$ & $1.81-11.20$ & 67 \\
VAS, pain score, low to high 0-10 cm & $6.6 \pm 2.7$ & $1-10$ & 37 \\
Physical capacity score, low to high 0-100 & $41.5 \pm 12.1$ & $9-63$ & 45 \\
Mental capacity score, low to high 0-100 & $37.2 \pm 13.3$ & $3-62$ & 45 \\
Bone mineral density, lumbar spine, g & $1065 \pm 120$ & $778-1367$ & 67 \\
Bone mineral density, right hip, $g$ & $961 \pm 110$ & $688-1222$ & 59 \\
Bone mineral density, left hip, g & $968 \pm 119$ & $705-1236$ & 59 \\
Bone mineral density, mean hip, g & $970 \pm 110$ & $705-1222$ & 61 \\
\hline
\end{tabular}

respectively. The oldest woman, 56 years, had osteoporosis and reported a wrist fracture.

Vitamin D deficiency, serum 25(OH)D $<25 \mathrm{nmol} / 1$ $(<10 \mathrm{ng} / \mathrm{ml})$ was found in $73 \%$, and only 4 women $(6 \%)$ had 25(OH)D >50 nmol/1 (Table 1). Mean S-PTH was 5.60土 1.98 , median $5.18 \mathrm{pmol} / 1$ and reference levels 1.60 $6.90 \mathrm{pmol} / 1$. Mean S-calcium was $2.32 \pm 0.09$, median $2.32 \mathrm{mmol} / \mathrm{l}$, reference levels $2.15-2.50 \mathrm{mmol} / \mathrm{l}$; mean serum ionized calcium was $1.23 \pm 0.04$, median $1.23 \mathrm{mmol} / 1$, reference levels $1.18-1.31 \mathrm{mmol} / \mathrm{l}$; and mean serum bone-specific ALP was 14.7 \pm 5.6 , median $13.5 \mu \mathrm{g} / \mathrm{l}$, reference values $2.9-$ $14.3 \mu \mathrm{g} / \mathrm{l}$ (Table 1).

There was no correlation between BMD at any region of interest and age, serum 25(OH)D, number of years in Sweden, parity, pain according to VAS or physical or mental capacity score according to SF-36, respectively.

\section{Discussion}

BMD was lower in the present Somali women than in the African-American referents for LUNAR DXA. BMD of the lumbar spine was also lower than in the white American referents. Vitamin D deficiency was frequent. However, no relation between BMD and duration of stay in Sweden or in relation to vitamin D levels was observed. Our results confirm a previous study with lower BMD of the forearm in a group of immigrant women in Finland at similar latitude [11]. The present results show that BMD was lower also at weightbearing regions at the lumbar spine and hips. Fractures were rare in these fairly young women.

The Somali women of the present study scored pain of a fairly high degree, but there was no relation between BMD and reported pain according to VAS. Poor muscle strength, vague musculoskeletal pain and low BMD are some of the clinical manifestations of hypovitaminosis D [12]. DXA application to detect osteomalacia is inconclusive, and a bone biopsy may be mandatory for histological diagnosis [13]. At higher ages, after the menopause, more women might precipitate osteoporosis, i.e. more BMD loss, during DXA measurement. Somali dietary habits, with less frequent intake of food containing vitamin D and calcium, wearing long robes and dresses, avoidance of sun exposure [14], having dark skin and living at northern latitudes [15] might contribute to the vitamin D deficiency.

There is a strong evidence showing that vitamin $\mathrm{D}$ plays a vital role in calcium metabolism, leading to bone ossification and bone remodelling and, hence, to maintain the BMD [1]. The present Somali women had lower spinal BMD in comparison to white women. This is in concordance with a study in the USA involving Somali immigrant women, AfricanAmerican women and white women, which showed higher 

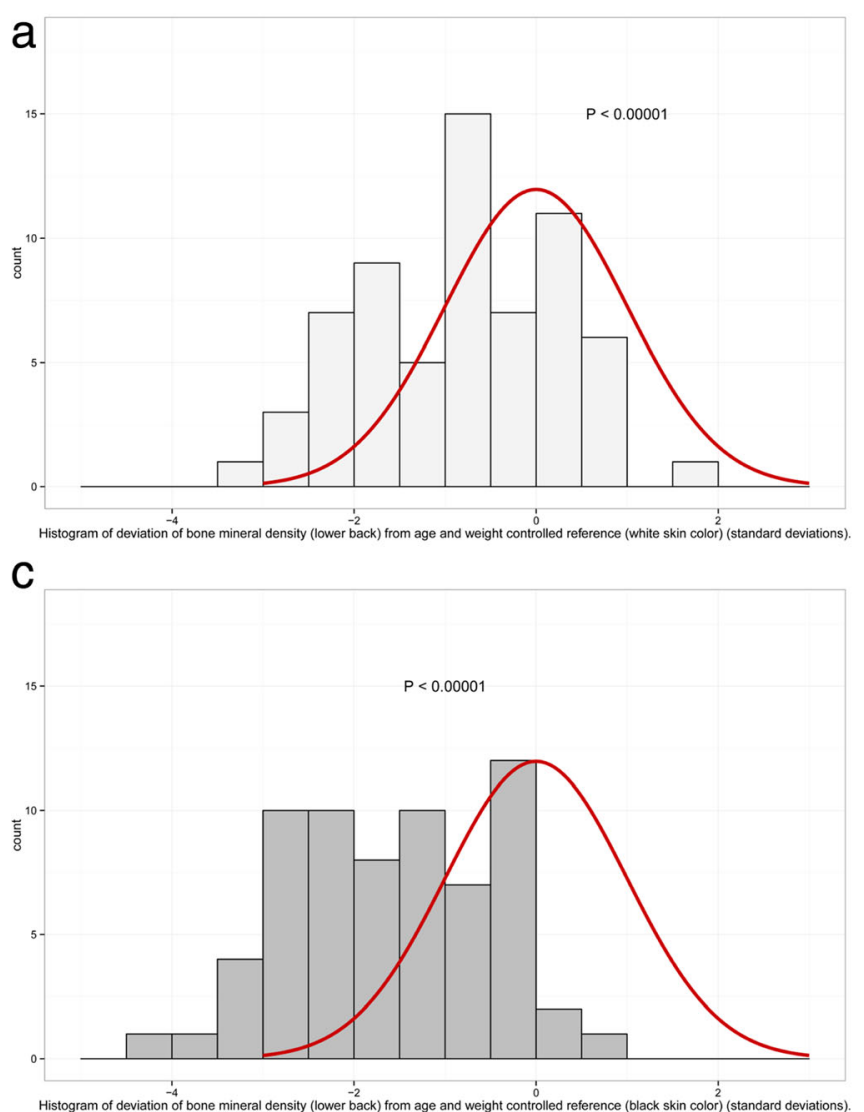

Fig. 1 a to d Histogram of bone mineral density (BMD) as Z-score (SD from the reference mean) in 67 Somali, premenopausal women compared with white American, lumbar spine (a), mean of right and left hip (b), and compared with African-American women, lumbar spine (c), mean of

spinal BMD value in African-American women than in both Somali and white women [9]. Somali immigrants in the USA and Somalis living in Finland did not exhibit the same robust BMD value compared with African-American women despite sharing the same African ancestry [11]. The possibility of genetic influences on BMD favouring the African-American group has also been discussed [16].

Low BMD defined as osteoporosis is a strong predictor for fractures [17]. Low BMD for age was found in $39 \%$ of the fairly young and premenopausal Somali women of this study. In Sweden, osteoporosis-linked fractures and morbidity incur a substantial medical expenditure and loss of quality of life [18].

The prevalence of osteoporosis in minority population is not yet explored in Sweden. Somalis are among the fastest growing groups of foreign-born residents in Sweden. Increased morbidity related to osteoporosis and fracture might incur a significant strain on the healthcare system in Sweden in the future when these women reach the postmenopausal stage and the fracture ages.

No correlation could be seen between BMD and duration of stay in Sweden. However, a larger sample might be needed
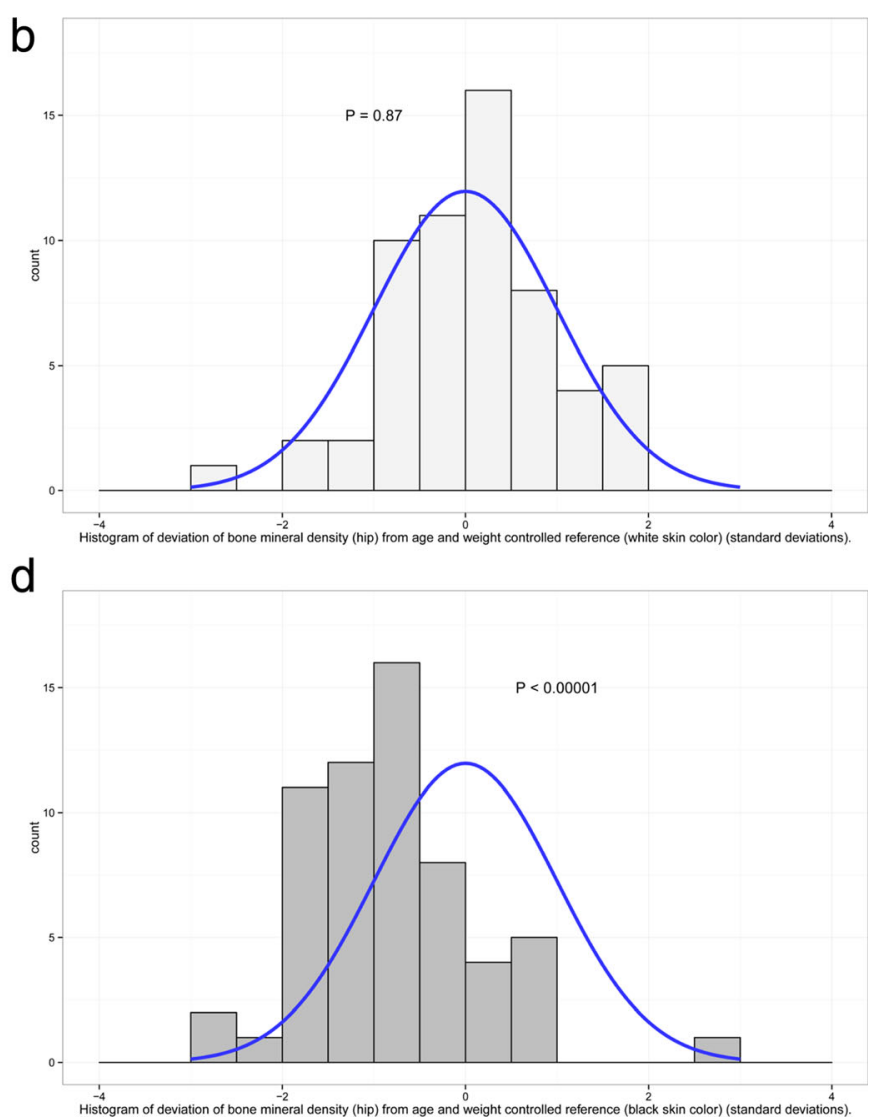

right and left hip (d), according to LUNAR dual energy X-ray absorptiometry (DXA) device reference populations. Count=number of subjects in each bin. The curves indicate the normal distribution according to the manufacturer's reference population

to reach a significant correlation. The mean duration of years in Sweden was 14 years which must be considered as long. Other factors as physical inactivity might be an even stronger predictor for BMD in this group of women. The degree of physical activity in native Swedish women correlated positively with S-25(OH)D, independently of other factors, in a random population sample of similar age in Gothenburg, as the present Somali women [19]. Physical activity is connected with being outdoors and thereby an increased sun exposure. There are reports of a lower degree of physical activity and staying indoors to a large extent in immigrant women at higher latitudes $[15,20]$ and could to some extent explain the lower BMD in Somali women living in Sweden.

Hypovitaminosis $\mathrm{D}$ was extensive in this study group, with $73 \%$ having deficiency. The majority also had elevated SPTH or in the upper reference interval, and serum bonespecific ALP levels were high. This suggests the need for supplementation to avoid demineralisation and further BMD loss and fractures. Encouraging the use of vitamin D supplements and following physician recommendations are emphasized. Information is important, and it was shown that 
immigrants with longer stay in their new country had a better awareness of osteoporosis and prevention [20].

One limitation was that bone biopsy was not performed for a definite diagnosis of osteomalacia. The procedure can be cumbersome and uncomfortable for the participants and difficult to motivate, making recruitment endeavours of such large group unattainable. A higher number of participants would probably be necessary to get statistical power for BMD correlations with duration of stay in Sweden. Age-matched native controls from Sweden had been of value. However, no comparisons could then have been performed with subjects of similar skin photo type. The LUNAR's African-American DXA reference population was based on BMD measures of women from Egypt according to the manufacturer.

A study strength was the engaging of a "hard to reach" group in research. This is often very difficult. Against all odds, we were able to recruit a fairly large number of premenopausal Somali women. It is also important to have only one national entity as genetic differences are found regarding BMD depending on the origin. BMD measurements were performed at both regions of interest, lumbar spine and hips and not limited to forearm, unlike other studies. An attempt was also made to evaluate the BMD relation to duration in Sweden, to parity and to degree of pain.

In conclusion, lumbar spine BMD in Somali women living in Sweden was lower than that in the white American DXA reference population. BMD on all regions of interest were lower than the African-American DXA referents. Vitamin D deficiency was common, and supplementation is advised to prevent from osteomalacia, osteoporosis and future fractures.

Acknowledgments The excellent work by BMA Stella Nakate is gratefully acknowledged. Grants were received from the ALF agreement at the Sahlgrenska University Hospital, the Swedish Heart Lung Foundation, the Swedish Council for Working Life and Social Research, the Regional Health Authority of West Sweden, the Swedish Medical Association and Gothenburg's Medical Association.

\section{Conflict of interest None.}

Open Access This article is distributed under the terms of the Creative Commons Attribution License which permits any use, distribution, and reproduction in any medium, provided the original author(s) and the source are credited.

\section{References}

1. Wacker M, Holick MF (2013) Vitamin D - effects on skeletal and extraskeletal health and the need for supplementation. Nutrients 5: $111-148$

2. Grant WB, Holick MF (2005) Benefits and requirements of vitamin D for optimal health: a review. Altern Med Rev 10:94-111
3. Yoshida T, Stern PH (2012) How vitamin D works on bone. Endocrinol Metab Clin N Am 41:557-569

4. Holick MF (2013) Bioavailability of vitamin D and its metabolites in black and white adults. N Engl J Med 369:2047-2048

5. Leslie WD (2012) Clinical review: ethnic differences in bone massclinical implications. J Clin Endocrinol Metab 97:4329-4340

6. Finkelstein JS, Lee M-LT, Sowers M, Ettinger B, Neer RM, Kelsey JL, Cauley JA, Huang MH, Greendale GA (2002) Ethnic variation in bone density in premenopausal and early perimenopausal women: effects of anthropometric and lifestyle factors. J Clin Endocrinol Metab 87:3057-3067

7. Gong G, Haynatzki G, Haynatzka V, Kosoko-Lasaki S, Howell R, Fu Y-X, Gallagher JC, Wilson MR (2006) Bone mineral density of recent African immigrants in the United States. J Natl Med Assoc 98: 746-752

8. Schnitzler CM (1993) Bone quality: a determinant for certain risk factors for bone fragility. Calcif Tissue Int 53(Suppl 1):S27-S31

9. Melton III LJ, Marquez MA, Achenbach SJ, Tefferi A, O'Connor MK, O'Fallon WM, Riggs BL (2002) Variations in bone density among persons of African heritage. Osteoporos Int 13:551-559

10. Sullivan M, Sullivan M, Karlsson J, Ware J (1995) The Swedish SF36 Health Survey-I. Evaluation of data quality, scaling assumptions, reliability and construct validity across general populations in Sweden. Soc Sci Med 41:1349-58

11. Islam MZ, Viljakainen HT, Kärkkäinen MU, Saarnio E, Laitinen K, Lamberg-Allardt C (2012) Prevalence of vitamin D deficiency and secondary hyperparathyroidism during winter in pre-menopausal Bangladeshi and Somali immigrant and ethnic Finnish women: associations with forearm bone mineral density. Br J Nutr 107:277-283

12. Mytton J, Frater AP, Oakley G, Murphy E, Barber MJ, Jahfar S (2007) Vitamin D deficiency in multicultural primary care: a case series of 299 patients. Br J Gen Pract 57:577-579

13. Bhan A, Rao AD, Rao DS (2012) Osteomalacia as a result of vitamin D deficiency. Rheum Dis Clin N Am 38:81-91

14. Benson J, Skull S (2007) Hiding from the sun—vitamin D deficiency in refugees. Aust Fam Physician 36:355-357

15. Lips P (2010) Worldwide status of vitamin D nutrition. J Steroid Biochem Mol Biol 121:297-300

16. Graafmans WC, Lips P, Ooms ME, van Leeuwen JP, Pols HA, Uitterlinden AG (1997) The effect of vitamin D supplementation on the bone mineral density of the femoral neck is associated with vitamin D receptor genotype. J Bone Miner Res 12:1241-1245

17. Johnell O, Kanis JA, Oden A, Johansson H, De Laet C, Delmas P, Eisman JA, Fujiwara S, Kroger H, Mellström D, Meunier PJ, Melton LJ 3rd, O'Neill T, Pols H, Reeve J, Silman A, Tenenhouse A (2005) Predictive value of BMD for hip and other fractures. J Bone Miner Res 20:1185-1194

18. Borgström F, Zethraeus N, Johnell O, Lidgren L, Ponzer S, Svensson O, Abdon P, Ornstein E, Lunsjö K, Thorngren KG, Sernbo I, Rehnberg C, Jönsson B (2006) Costs and quality of life associated with osteoporosis-related fractures in Sweden. Osteoporos Int 17: $637-650$

19. Landin-Wilhelmsen K, Wilhelmsen L, Wilske J, Lappas G, Rosen T, Lindstedt G, Lundberg P-A, Bengtsson B-Å (1995) Sunlight increases serum $25(\mathrm{OH})$ vitamin $\mathrm{D}$ concentration whereas 1 , $25(\mathrm{OH}) 2 \mathrm{D} 3$ is unaffected. Results from a general population study in Gothenburg, Sweden. (The WHO MONICA Project). Eur J Clin Nutr 49:400-407

20. Tan S, Ji L, Tsai J, Eng J, Ko HJ, Yau A, Edwards G, Bunta A, Edwards BJ (2009) Greater osteoporosis educational outreach is desirable among Chinese immigrants in Chinatown. Chic Osteoporos Int 20:1517-1522 